This Section of Epidemiology and Psychiatric Sciences appears in each issue of the Journal to stress the role of the epidemiological approach to promote advances in the field of clinical psychopharmacology, with a particular attention to controversial findings. The ultimate aims are to help develop a more critical attitude towards the results of research studies published in the international literature, to promote original research projects with higher methodological standards, and to implement the most relevant results of research in every-day clinical practice. These contributions are written in house by the journal's editorial team or commissioned by the Section Editor (no more than 1000 words, short unstructured abstract, 4 key-words, one Table or Figure and up to ten references).

Corrado Barbui, Section Editor

\title{
Can a digital medicine system improve adherence to antipsychotic treatment?
}

\author{
D. Papola*, C. Gastaldon and G. Ostuzzi
}

Department of Neuroscience, Biomedicine and Movement Sciences, Section of Psychiatry, University of Verona, Verona, Italy

A substantial proportion of people with mental health conditions do not adhere to prescribed pharmacological treatments. Poor adherence is probably one of the most critical elements contributing to relapse in people with schizophrenia and other severe mental disorders. In order to tackle this global issue, in November 2017 the Food and Drug Administration approved a tablet formulation of the atypical antipsychotic aripiprazole embedded with a novel digital adherence-assessment device. In this commentary, we critically appraised the potential beneficial and harmful consequences of this new digital formulation of aripiprazole, and we highlighted expected implications for clinical practice.

Received 1 February 2018; Accepted 1 February 2018; First published online 12 March 2018

Key words: Antipsychotics, evidence-based psychiatry, pharmacological marketing, psychosis.

Improving adherence to prescribed medicines is key in healthcare, as recently emphasised by the World Health Organization (WHO, 2003). Among people with severe mental disorders, those suffering from schizophrenia and bipolar disorder have particularly high levels of non-adherence, which contributes to the high burden of disability. Indeed, it has been estimated that only one out of three patients with schizophrenia is fully adherent to antipsychotic treatment and that at least $50 \%$ of patients are not fully adherent with their medications at some time during the treatment (Marder, 2003). It is worth noting that, among other factors, medication non-adherence is a relevant predictor of relapse (Chen et al. 2005).

\footnotetext{
* Address for correspondence: Dr Davide Papola, Section of Psychiatry, University of Verona, Ospedale Policlinico GB Rossi, Piazzale L.A. Scuro, 10 - 37134 Verona, Italy. (E-mail: candido09@hotmail.it)
}

Among the strategies that community psychiatric services can implement to reduce medication nonadherence in patients with severe mental disorders there are pill counts, electronic devices that capture pill container openings such as the Medication Event Monitoring System (MEMS), bottle caps, pharmacy refill records, mobile phone text reminders, psychosocial interventions such as 'adherence therapy', and even financial reinforces (Nieuwlaat et al. 2014). Additionally, long-acting injectable antipsychotics (LAIs) are another frequently used approach to address non-adherence. However, LAIs have been largely criticised for their limited flexibility in dosage control, the pain on the injection site and the culturally rooted idea of them as stigmatising and coercive drugs (Kirschner et al. 2013). By contrast, LAIs may notably simplify the daily routine of the patient, reduce the need of control by family members, and can ultimately ameliorate subjective attitude towards medications, and overall quality of life (Das et al. 2014). 
A novel medication adherence-assessment device, called digital medicine system (DMS), has recently been designed to assist health-care providers in objectively assessing patient adherence to prescribed medicines. The DMS electronically confirms adherence to oral medication, acquires physiologic metrics periodically and remotely, and presents this information in a centralised manner. Although the DSM technology has been mostly tested in individuals with physical diseases (Frias et al. 2017), the first digital medicine released on the market is an antipsychotic. On November 13, 2017 the Food and Drug Administration (FDA) approved 'Abilify MyCite' as the first drug with a digital ingestion tracking system.

Abilify MyCite is a tablet formulation of the atypical antipsychotic aripiprazole embedded with a small edible sensor $(1 \mathrm{~mm} \times 1 \mathrm{~mm})$. After the ingestion of the digital medicine, acid fluids of the stomach activate a signal, which is detected by a wearable sensor placed on the chest. The sensor forwards data to an application on the patient's smartphone, which sends data to a cloud-based server. Caregivers and physicians can this way access, through a web-based portal, information on medicine use. Abilify MyCite is indicated in the treatment of schizophrenia, in the acute treatment of manic and mixed episodes associated with bipolar I disorder, and as an add-on treatment for depression in adults. As soon as Abilify MyCite was submitted to the FDA approval process in September 2015, the CEO of Otsuka Pharmaceutical industry called for a 'revolution of the way adherence is measured' (Carson, 2015). The aim of this commentary is to critically discuss how Abilify MyCyte may improve treatment adherence, including its potential advantages and shortcomings.

To date, a handful of studies were conducted to shade light on the feasibility of utilising a DMS to electronically confirm ingestion of oral medications using networked sensors in people suffering from severe mental disorders. The first is an observational pilot investigation that enrolled 28 patients with schizophrenia or bipolar disorder and demonstrated that the networked DMS yielded high detection accuracy when compared with directly observed ingestion, and was well tolerated and accepted by patients (Kane et al. 2013). Another study, including 67 patients with schizophrenia, yielded similar results, demonstrating also the ability of the majority of patients to use the digital medicine device with good satisfaction, independently or with minimal assistance (Peters-Strickland et al. 2016). An 8-week, singe-arm trial that enrolled 49 patients diagnosed with schizophrenia, bipolar or depressive disorder suggested that an integrated call centre can optimise the use of the DMS (Kopelowicz et al. 2017).

A challenging aspect of these studies may be selection bias, as the population enrolled was composed by individuals who were motivated to participate in the study and to use such a device. Consequently, participants may have been more likely to adhere to the treatment received and possibly more willing to tolerate minor adverse effects (such as erythema or contact dermatitis related to the wearable sensor implant). Furthermore, although participants had a diagnosis of schizophrenia, they had a relatively low level of impairment. Thus, these preliminary studies may have overemphasised the beneficial effects of Abilify Mycite, including its practicality of use in people with severe mental disorders.

Additional important aspects are costs, patient values and preferences. To date, information on the cost of Abilify Mycite on a monthly basis is not available. This hampers the possibility to assess its cost-effectiveness. In term of patient values and preferences, a first consideration is that this device may complicate the patient daily routine, as they should not only remember to take the medication but also deal with all the possible everyday setbacks related to the patch stuck on the chest, such as remembering to change it every week, adherence problems in case of sweating, the possible need to depilate the skin for better adherence. Considering that patients with low illness insight often struggle to comply with relatively simple therapeutic regimes, how can they adhere to such a complex routine? Second, among reasons for refusing medications, perceived coercion and control is often reported and one may argue that this device may likely worsen these subjective feelings, particularly in patients with high levels of suspiciousness, paranoid thoughts and, most of all, experiences passivity and external control of thoughts or body functions. Paradoxically, only patients with good insight and adherence, who would probably not need an electronic monitoring, are most likely to comply with all the requirements related to Abilify Mycite. Third, it is not clear whether the function of the sensor in the pill can be somehow manipulated (for example, by melting the tablet in the water or other liquids).

These considerations lead to the conclusion that while the DMS technology demonstrated the technical capacity to detect and monitor tablet ingestion with high accuracy, acceptable latency and good tolerability, it remains unclear whether this effect may translate into improved adherence in real-world settings. Studies comparing Abilify Mycite with LAIs or other strategies aimed at improving treatment adherence should be conducted to shed light on these issues, and to ascertain its impact on patients' drug attitude and quality of life. In the meantime, considering that 'the ability of Abilify MyCite to improve treatment adherence has not been established' (Otsuka Pharmaceutical Co. 2017), and that the technology 
may cast a spooky shadow on the privacy rights of people (Belluck, 2017), it seems difficult to recommend it for everyday clinical practice.

\section{Financial support}

No financial support was received for this paper from any funding agency, commercial or not-for-profit sectors.

\section{Conflict of interest}

None.

\section{References}

Belluck P (2017). First Digital Pill Approved to Worries About Biomedical 'Big Brother'. The New York Times. Retrieved 30 November 2017 from https://www.nytimes. com/2017/11/13/health/digital-pill-fda.html.

Carson WH (2015). Abilify MyCite - U.S. FDA Accepts First Digital Medicine New Drug Application for Otsuka and Proteus Digital Health. Retrieved 5 December 2017 from https://www.drugs.com/nda/abilify_digital_150910.html.

Chen EY, Hui CL, Dunn EL, Miao MY, Yeung WS, Wong CK, Chan WF, Tang WN (2005). A prospective 3-year longitudinal study of cognitive predictors of relapse in first-episode schizophrenic patients. Schizophrenia Research 77, 99-104.

Das AK, Malik A, Haddad PM (2014). A qualitative study of the attitudes of patients in an early intervention service towards antipsychotic long-acting injections. Therapeutic Advances in Psychopharmacology 4, 179-185.

Frias J, Virdi N, Raja P, Kim Y, Savage G, Osterberg L (2017). Effectiveness of digital medicines to improve clinical outcomes in patients with uncontrolled hypertension and type 2 diabetes: prospective, open-label, cluster-randomized pilot clinical trial. Journal of Medical Internet Research 19, e246.
Kane JM, Perlis RH, DiCarlo LA, Au-Yeung K, Duong J, Petrides G (2013). First experience with a wireless system incorporating physiologic assessments and direct confirmation of digital tablet ingestions in ambulatory patients with schizophrenia or bipolar disorder. Journal of Clinical Psychiatry 74, e533-e540.

Kirschner M, Theodoridou A, Fusar-Poli P, Kaiser S, Jager M (2013). Patients' and clinicians' attitude towards long-acting depot antipsychotics in subjects with a first episode of psychosis. Therapeutic Advances in Psychopharmacology 3, 89-99.

Kopelowicz A, Baker RA, Zhao C, Brewer C, Lawson E, Peters-Strickland T (2017). A multicenter, open-label, pilot study evaluating the functionality of an integrated call center for a digital medicine system to optimize monitoring of adherence to oral aripiprazole in adult patients with serious mental illness. Neuropsychiatric Disease and Treatment 13, 2641-2651.

Marder SR (2003). Overview of partial compliance. Journal of Clinical Psychiatry 64(Suppl. 16), 3-9.

Nieuwlaat R, Wilczynski N, Navarro T, Hobson N, Jeffery R, Keepanasseril A, Agoritsas T, Mistry N, Iorio A, Jack S, Sivaramalingam B, Iserman E, Mustafa RA, Jedraszewski D, Cotoi C, Haynes RB (2014). Interventions for enhancing medication adherence. Cochrane Database of Systematic Reviews 11, CD000011. doi: 10.1002/14651858.CD000011.pub4.

Otsuka Pharmaceutical Co. (2017). Abilify MyCite's prescribing information (labeling) notes. Retrieved 2 December 2017 from https://www.accessdata.fda.gov/ drugsatfda_docs/label/2017/207202lbl.pdf

Peters-Strickland T, Pestreich L, Hatch A, Rohatagi S, Baker RA, Docherty JP, Markovtsova L, Raja P, Weiden PJ, Walling DP (2016). Usability of a novel digital medicine system in adults with schizophrenia treated with sensor-embedded tablets of aripiprazole. Neuropsychiatric Disease and Treatment 12, 2587-2594.

WHO (2003). Adherence to Long-Term Therapies: Evidence for Action. Geneva: World Health Organization. Retrieved 2 December 2017 from http://www.who.int/chp/knowledge/ publications/adherence_report/en/. 